\title{
Música, movimiento y danza: un enfoque integrador para la formación inicial del profesorado
}

\section{Music, Movement, and Dance: an Integral Approach for Initial Teacher Training}

\author{
Silvia Sánchez Ariño*1 \\ silvia.sanchez@udg.edu \\ Dolors Cañabate Ortiz* \\ dolors.canyabate@udg.edu \\ Montserrat Calbó Angrill* \\ montserrat.calbo@udg.edu \\ ISABEL Viscarro Tomás** \\ isabel.viscarro@urv.cat \\ * Universidad de Girona, España \\ ** Universidad Rovira y Virgili, España
}

\section{Resumen:}

La consideración del cuerpo y del movimiento como medios de expresión y comprensión musical tendría que formar parte de una educación musical activa y vivencial. El estudio que se ha llevado a término describe cómo se integra y desarrolla el trabajo del movimiento y la danza en las sesiones de educación musical de 19 escuelas a partir de las observaciones e intervenciones de estudiantes de Magisterio Musical en prácticas. Las conclusiones del estudio apuntan a que, si bien hay una cierta integración del movimiento y la danza en la educación musical, es necesario mejorar aspectos como el del trabajo de la improvisación y la composición corporal, ambos instrumentos imprescindi-

\begin{abstract}
:
The consideration of the body and movement as ways of expressing and understanding music should be part of an active musical and experiential education. The following study describes how the work of movement and dance adapts and develops in the music education sessions of 19 schools from observations and interventions made by prospective teachers of music during their school training. The findings of the study suggest that, although there is some integration of movement and dance in music education, it is necessary to improve aspects such as improvisation and body composition, which are both essential tools for the development of students' creative and communicative abilities.
\end{abstract}

1 Dirección para correspondencia (correspondence address):

Silvia Sánchez Ariño. Departamento de Didácticas Específicas. Facultad de Educación y Psicología. Universidad de Girona. Plaça Sant Domènec, 9. 17071 Girona (España). 
Música, movimiento y danza: un enfoque integrador para la formación inicial del profesorado

Silvia Sánchez Ariño, Dolors Cañabate Ortiz, Montserrat Calbó Angrill e Isabel Viscarro Tomás

bles para el desarrollo de las capacidades creativas y comunicativas del alumnado.

\section{Palabras clave:}

Educación musical; Educación física; Danza; Practicum; Formación inicial de maestros/as; Educación basada en competencias.

\section{Key words:}

Music Education; Physical Education; Dance; Practicum; Initial Teacher Training; Competency-Based Education.

\section{Résumé:}

Musique, Mouvement et Danse: Une approche intégrée de la formation initiale des enseignants.

$\mathrm{L}^{\prime}$ analyse du corps et du mouvement comme moyen d'expression et la compréhension musicale devrait faire partie d'une éducation musicale active et expérimentée. L étude qui a été menée à terme décrit comment s`adapte et se développe le travail du mouvement et de la danse lors des séances diéducation musicale de dix-neuf écoles à partir des observations et interventions des étudiants en enseignement musical au cours de la pratique. Les résultats de liétude suggèrent que, bien qusil y ait intégration du mouvement et de la danse dans lienseignement de la musique, il est nécessaire diaméliorer certains aspects tels que le travail dıimprovisation et de composition corporelle; deux outils essentiels au développement des aptitudes créatives et communicatives des élèves.

\section{Mots clés:}

Éducation musicale; éducation physique; danse; stage; formation initiale des enseignants; éducation axée sur les compétences.

Fecha de recepción: 3-11-2013

Fecha de aceptación: 30-4-2014

\section{Introducción}

La consideración del cuerpo y del movimiento como medios de expresión y comprensión musical ha formado parte de los métodos activos de educación musical del siglo XX más relevantes. El origen de esta idea lo encontramos en el método de educación musical La Rítmica, creado por el compositor y pedagogo suizo Émile Jaques-Dalcroze (1865-1950), que influyó decisivamente en la pedagogía musical y estableció las bases para un uso educativo y reeducador de la música y el movimiento. En el camino de adaptación y actualización que ha seguido, la educación rítmica ha probado de manera empírica, tanto en sus aportaciones a la educación musical como a la educación general, su valor en el desarrollo de las facultades artísticas del ser humano (Del Bianco, 2009).

Debemos a Jaques-Dalcroze, por tanto, haber demostrado que la música no la escucha sólo el oído sino todo el cuerpo, por lo que «el aprendizaje de la música supone, en consecuencia, la posibilidad de sentir y 
representar el movimiento que encierra" (Bachmann, 1998:27). Cualquiera de los elementos que conforman la música -la pulsación, el fraseo, el ritmo, el espacio melódico y armónico, la forma, la articulación, el carácter o la textura- pueden ser abordados a partir del movimiento corporal y materializarse en el espacio que nos rodea. El trabajo corporal consciente del fenómeno sonoro creará imágenes sensoriales (musculares y visuales) que posibilitarán una vivencia y una comprensión más consciente e intensa de la música.

Otra idea que nos lleva a considerar la importancia del movimiento en la educación musical es la que señala Bachmann: "La música del siglo XX, a partir de Debussy, ha consistido en gran parte en explotar, a veces sistemáticamente, los recursos espaciales del mundo sonoro. En la música actual, más que nunca, tiempo y espacio adquieren sentido el uno gracias al otro. La mirada que dirigimos a la música del pasado se ve así renovada y enriquecida. Porque cuando se la considera en toda su extensión es cuando la música revela lo que nunca ha dejado de ser: arte a la vez del espacio y del tiempo "(Bachmann, 1998: 38). A partir de esta reflexión y considerando que el movimiento corporal no sólo es la fuente de todas nuestras manifestaciones, nuestro principal medio de expresión y de relación con los demás, sino también nuestra forma personal de ocupar el espacio, "el maestro de música que quiere hacer comprender a sus alumnos la música en toda su "espacialidad " no podría, pues, apelar a mejores modelos que los que le ofrece el movimiento corporal" (Bachmann, 1998: 38). Por otra parte, la música, como manifestación de las relaciones sonoras y espacio-temporales, presenta total correspondencia no sólo con el movimiento sino también con la danza en tanto que ésta puede representar la vivencia corporal de la música. De hecho, la correspondencia música-danza supone seguramente la sinestesia más antigua y fructífera de la historia de las manifestaciones artísticas. Es lógico, por tanto, que la pedagogía musical incorpore la rítmica y la danza como elementos básicos del aprendizaje musical. La danza y el movimiento expresivo, son dos dimensiones interpretativas importantes de la música, las cuales poseen un gran valor educativo (Martín Escobar, 2005)

En el currículo de educación primaria de Cataluña derivado de la LOGSE se reflejaban parte de los planteamientos expuestos ahora en la LOE. El área de educación artística (englobada por las materias de visual y plástica y de música) seleccionaba contenidos relacionados con el mo- 
vimiento y la danza para la enseñanza y aprendizaje de la música, contenidos que también estaban presentes en el área de educación física.

La danza aporta a la Educación Física la adquisición y desarrollo de las habilidades y destrezas básicas, así como el desarrollo de la coordinación y de las habilidades perceptivo-motoras (Gregorio y cols., 2010). Además es un agente educativo dentro de la expresión corporal que permite una educación del cuerpo y del movimiento. Permite el conocimiento de uno mismo, el de otras culturas, el desarrollo de la creatividad y de la capacidad expresiva entre otros aspectos (García y cols., 2011). A pesar de reconocer por diversos autores que la danza es un buen recurso educativo y didáctico a tener en cuenta para el planteamiento y el buen desarrollo de las competencias introducidas en el currículo escolar (Pedrero, 2013), existen diversos estudios donde se llega a la conclusión de que esta no es tratada con asiduidad concretamente en el área de Educación física por diferentes aspectos, principalmente culturales e históricos (Pérez-Castro y Urdampilleta, 2012).

Estamos de acuerdo con cuando dice que la danza debe ser un recurso educativo y didáctico a tener en cuenta para el planteamiento y el buen desarrollo de las competencias introducidas en el currículo escolar.

Respecto a la enseñanza de la danza, básicamente tradicional catalana, la recomendación de los expertos para llevarla a la práctica era con la interrelación de los maestros y maestras de educación física, los y las maestras de educación musical y de los y las maestras tutoras, dada la situación actual de las escuelas en cuanto a recursos humanos (Pujol y Serra, 1998). Actualmente el nuevo currículo de educación primaria en Cataluña, estructura el área de educación artística en dos bloques de contenidos relacionados con dos ámbitos de la experiencia estética: explorar y percibir e interpretar y crear. Cada uno de estos ámbitos agrupa los contenidos en los apartados de visual y plástica y de música y danza. Se dice que la inclusión del lenguaje plástico y del lenguaje musical en una sola área debe permitir un enfoque globalizado que incluya las estrechas conexiones entre los diferentes lenguajes y la incorporación de contenidos de danza y teatro. Por otro lado, se señala que el área de educación artística mantiene unos enlaces evidentes con el área de educación física, en tanto que ambas áreas trabajan el sentido estético y creativo de la expresión y comunicación corporal, de manera especial por medio de la danza. Además, hay que tener presente que la finalidad central de cada una de las áreas curriculares debe ser el desarrollo de las 
competencias básicas de manera que se conviertan en el eje vertebrador del proceso educativo.

\section{Planteamiento del problema y objetivos}

El estudio que se ha llevado a cabo quiere describir cómo se integra y desarrolla el trabajo del movimiento y la danza en las sesiones de educación musical de 19 escuelas de educación infantil y primaria a partir de las observaciones e intervenciones de estudiantes de tercer curso en prácticas de los estudios de Maestro-a en Educación Musical de la Universidad de Girona.

Por otro lado, el estudio pretende también contribuir a que los estudiantes desarrollen una capacidad de observación de la actividad docente más estructurada, reflexiva y crítica y a que mejoren la relación e integración de los conocimientos teórico-prácticos y profesionales.

\section{Metodología}

Este estudio toma como fundamentación teórica la investigación didáctica descriptiva y explicativa (Schubauer-Leoni, 1998) de lo que Rickenmann (2006) Ilama la enseñanza ordinaria. Éste entiende por enseñanza ordinaria aquella que se desarrolla actualmente en la mayoría de las aulas, más allá de los dispositivos de innovación pedagógica, a partir de las prácticas corrientes de los profesores/profesoras.

En las "ciencias" didácticas está relativamente poco explorada la vía de una de una ciencia comprensiva, una didáctica no prescriptiva, que por una parte permita responder a contextos empíricos en los que se han desarrollado las observaciones, y por otra, descubrir relaciones inauditas entre objetos de investigación y modelos teóricos.

Situamos nuestra investigación como investigación educativa, basada en una metodología cualitativa e interpretativa, a partir de observaciones etnográficas, que pretende describir y analizar en términos cualitativos, pero también cuantitativos en cuanto los datos cuantificables pueden interesar para enfocar la situación de los parámetros estudiados en contextos escolares reales (las escuelas de las comarcas de Girona).

Nuestra investigación implica una observación directa dentro de un 
escenario educativo, observación que se refleja en una especie de cuaderno de campo pautado. En nuestra versión particular de la metodología etnográfica se da una intersección de lo puramente descriptivo (que podrá, en ocasiones, reforzarse en esta investigación mediante videograbaciones) y lo interpretativo, puesto que nuestros informantes son los estudiantes en prácticas y al escribir sus comentarios en las fichas de observación realizan por supuesto una primera interpretación de lo que observan y viven.

En este sentido, es importante remarcar que esta metodología tiene un fuerte componente de aprendizaje reflexivo para los futuros maestros, puesto que les permite a la vez observar, investigar y aprender, tejiendo sus propias observaciones con categorías aprendidas, deducciones argumentadas y conversaciones con los maestros observados.

\section{Muestra}

La muestra para llevar a cabo el estudio ha sido de 19 escuelas de educación infantil y primaria que acogen estudiantes en prácticas del tercer curso de maestro de educación primaria, mención de educación musical.

Se han observado un total de 902 sesiones de educación musical de los diferentes cursos del segundo ciclo de educación infantil y de los tres ciclos de educación primaria a lo largo del curso escolar 2011-2012.

\section{Instrumento para la recogida de información}

Las observaciones de las sesiones de clase se registraron a través de un registro sistemático (supone una cierta planificación previa, de manera formalizada en relación a lo que se observa, concretando el cómo y cuándo, según Coll y Onrubia, 1999) que se proporcionó a los estudiantes en prácticas y en la que debía constar: día de la sesión, nivel educativo, especialidad de la o el maestro que impartía la sesión o especificar si era una intervención del estudiante, breve descripción de la actividad de enseñanza y aprendizaje, contenidos trabajados (musicales, corporales, de danza, visuales y plásticos, de otras áreas ...), observaciones en que consideraban que durante el proceso de enseñanza y aprendizaje se 
incidía especialmente en el trabajo de la sensibilidad artística, metodología desarrollada y otros comentarios a aportar. Para poder contextualizar las observaciones también se recogieron algunos datos referentes al centro docente y a la enseñanza de la música y la danza en el centro. Las observaciones se llevaron a cabo durante nueve semanas del tercer y último período de prácticas que se realiza en el tercer curso de grado de maestro-a de primaria, por lo tanto, cuando la mayoría de los estudiantes estaba finalizando los estudios. Durante este período los estudiantes combinan su formación en la escuela y la facultad, lo que permitió poder ir revisando y resolviendo las dudas que surgían en la elaboración del trabajo de campo.

Todas las sesiones, tanto las de infantil como las de primaria, han sido impartidas por un o una maestra en educación musical excepto en una de las escuelas en la que han sido impartidas por una maestra especialista de educación especial. Algunas sesiones también han sido intervenciones de los practicantes de la mención de Música.

\section{Análisis de datos}

El primer análisis de los datos se ha hecho a partir de los siguientes criterios:

- Conocer la frecuencia en que se presenta el trabajo del movimiento y la danza en las sesiones de clase.

- Describir el tipo de actividad en que se integra el movimiento y la danza según las siguientes categorías: canción con gestos (cuando se gestualiza el texto de una canción), canción con movimiento (cuando se asocia a una canción algún movimiento con desplazamiento), canción danzada (cuando se canta y baila al mismo tiempo), audición y movimiento (cuando se trabaja algún elemento musical de una audición a través del movimiento), audición dramatizada (cuando se escenifica el argumento de una obra o fragmento musical), lenguaje musical y movimiento (cuando se trabaja algún elemento melódico, rítmico, formal, dinámico ... a través del movimiento), danza (cuando se trabaja el lenguaje de la danza) y producción artística (cuando se interpreta o elabora algún trabajo que integra diversos lenguajes artísticos a la vez).

- Describir el tipo de proceso artístico que caracteriza la actividad 
según las siguientes categorías: interpretación corporal o dancística (cuando el movimiento que ejecutan los niños y niñas está previamente establecido o cuando la coreografía de la danza ya está previamente creada), improvisación corporal (cuando los niños y niñas exploran en tiempo real algún tipo de movimiento, es decir, cuando hay simultaneidad entre la invención y la ejecución corporal) y composición coreográfica (cuando los niños y niñas elaboran alguna creación corporal a partir de un proceso más reflexivo y pautado).

La muestra inicial para llevar a cabo el estudio ha sido de 19 escuelas de educación infantil y primaria que acogen estudiantes en prácticas de tercer curso de Magisterio Musical. Después de tratar los datos obtenidos, se ha constatado que en una de las 19 escuelas seleccionadas el trabajo del movimiento y la danza en las sesiones de educación musical es casi inexistente y también que, durante el período en que se llevaron a cabo las observaciones, dos escuelas estaban inmersas en el trabajo de proyectos musicales que tampoco estaban relacionados con el movimiento o la danza. Por lo tanto, algunos de los resultados que se presentan corresponden a una muestra de 16 escuelas. Se han observado un total de 902 sesiones de educación musical de los diferentes cursos del segundo ciclo de educación infantil y de los tres ciclos de educación primaria. Todas las sesiones, tanto las de infantil como las de primaria, han sido impartidas por un o una maestra en educación musical excepto en una de las escuelas en la que han sido impartidas por una maestra especialista de educación especial. Algunas sesiones también han sido intervenciones de los practicantes de la Mención de música.

\section{Resultados y discusión}

De las 902 sesiones, en 430 se ha trabajado el movimiento o la danza, es decir, en un $47,67 \%$ del total de sesiones de educación musical.

El gráfico 1 muestra el número total de sesiones observadas en cada una de las escuelas y se especifica el número de sesiones que han incluido algún trabajo de movimiento o danza y el número de las que no lo han incluido. La diferencia con respecto al número total de sesiones de educación musical por escuelas se debe principalmente a que el horario 
de prácticas de algunos estudiantes también incluía sesiones de otras materias.

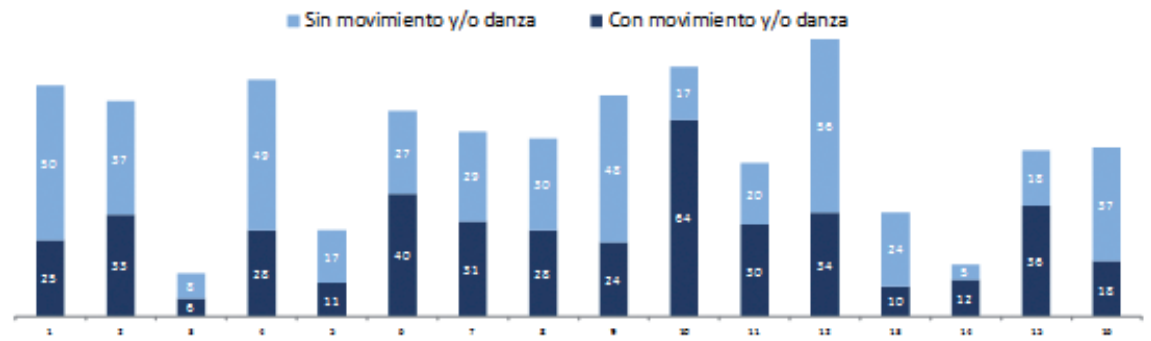

Gráfico 1. Número de sesiones de educación musical por escuelas que han incluido el movimiento o la danza y número de las que no lo han incluido

En el gráfico 2 se pueden ver los mismos resultados en forma de porcentajes. Haciendo una lectura global se evidencia una presencia moderada del movimiento y la danza teniendo en cuenta que el y la maestra de educación musical debe desarrollar tres tipos de actividades de expresión musical: vocal, instrumental y corporal.

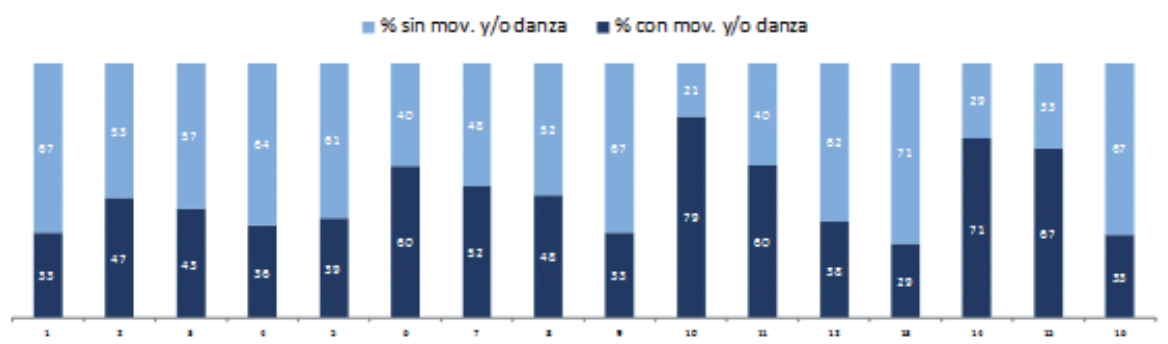

Gráfico 2. Número de sesiones de educación musical por escuelas que han incluido el movimiento o la danza y número de las que no lo han incluido en forma de porcentajes

Respecto a la tipología de actividades musicales con movimiento o danza (gráfico 3), a nivel global se evidencia una buena integración del movimiento y la danza en el trabajo de la canción, la audición y el lenguaje musical; esta vinculación del trabajo corporal en actividades musicales es una buena preparación para el trabajo específico de la danza que también muestra una presencia notable. La culminación de este traba- 
jo corporal y musical en actividades de elaboración de producciones artísticas que posibiliten la integración de otros lenguajes artísticos se desarrolla excepcionalmente.
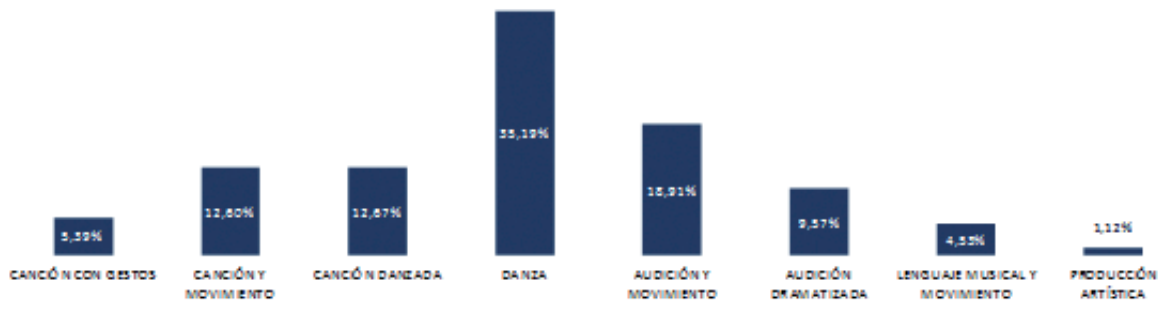

\section{Gráfico 3. Tipología de actividades con movimiento o danza}

En cuanto al tipo de proceso artístico (gráfico 4) se ha evidenciado un fuerte desequilibrio entre las actividades de interpretación corporal o dancística $(88,22 \%)$, de improvisación corporal $(7,09 \%)$ y de composición coreográfica $(4,69 \%)$. Sin quitar valor al acto de la interpretación, que de hecho es una recreación de una obra ya existente enriquecida con la sensibilidad del intérprete, es en la improvisación y la composición donde pueden aflorar con más fuerza las capacidades creativas del alumnado, además de que son procedimientos que deben estar presentes en el aprendizaje de cualquier lenguaje.
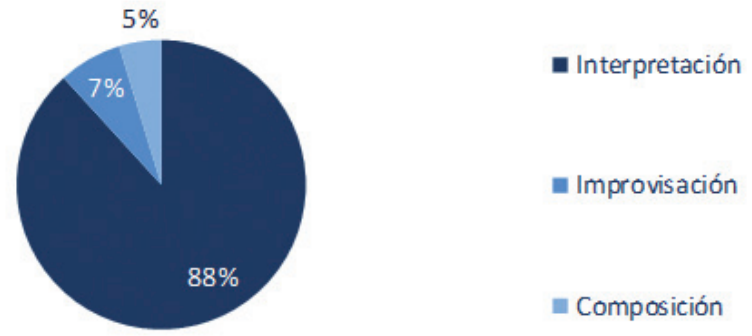

\section{Gráfico 4. Tipo de proceso artístico que caracteriza la actividad}

El análisis de los datos que se han recogido por escuelas y niveles educativos, que se presentará en un trabajo posterior, permitirá describir con más profundidad las singularidades de cada una de las realidades y prácticas educativas estudiadas. Respecto al trabajo en equipo de la danza entre las y los maestros en educación musical y las y los maestros en educación física, los resultados muestran que en 9 de las 19 
escuelas la danza sólo se trabaja desde el área de educación artística (en una de estas 9 escuelas el trabajo se hace puntualmente con los tutores para preparar la fiesta de la escuela), lo que indica una baja implicación del área de educación física. De las 10 escuelas que trabajan la danza desde las áreas de educación física y de educación artística se observa que: 4 escuelas trabajan de manera coordinada regularmente, 5 escuelas lo hacen de manera puntual (por la fiesta de carnaval, para celebrar el día de la danza, para un encuentro de danzas con otras escuelas o simplemente para decidir las danzas que trabajarán durante el curso) y 1 escuela trabaja la danza desde las dos áreas pero no de forma interdisciplinar.

Por último, los estudiantes han valorado positivamente la elaboración del trabajo y algunas de las aportaciones a su formación que destacan son que ha sido una buena herramienta para reflexionar y opinar sobre el tratamiento de la música, el movimiento y la danza en su centro de prácticas y para valorar su importancia en la educación de los niños y niñas; también señalan que han podido relacionar los diferentes aspectos curriculares relacionados con la música, el movimiento y la danza con la práctica educativa o que les ha permitido elaborar un archivo de propuestas, dinámicas y actividades útiles para la futura práctica profesional. También dicen que ha sido una oportunidad para llevar a término una observación más estructurada, analítica y crítica de la actividad docente y para autoevaluar la propia práctica. En definitiva, un buen ejercicio de práctica reflexiva que puede ayudar a comprender mejor la complejidad del hecho docente.

\section{Conclusiones}

Algunas de las conclusiones que se pueden extraer a partir de los primeros resultados obtenidos son que, aunque se constata una presencia moderada del trabajo del movimiento y la danza en las sesiones de educación musical, se pone de manifiesto que es necesaria una mayor implicación del área de educación física en el trabajo específico de la danza así como una mayor concienciación de la importancia de un trabajo coordinado de la misma entre las y los maestros especialistas de educación musical y de educación física. Este trabajo en equipo de los contenidos de danza que comparten las dos áreas puede aportar una 
mayor coherencia al aprendizaje del alumnado y puede favorecer el desarrollo de la capacidad para transferir aprendizajes.

Por otro lado, la lectura global de las prácticas docentes estudiadas muestra que el trabajo corporal se integra dentro de las actividades propiamente musicales como la canción, la audición o el lenguaje musical y también se evidencia un trabajo notable del lenguaje específico de la danza. En cambio, encontramos a faltar actividades de producción musical o artística (desarrollados evidentemente en equipo con otros docentes) que integren diferentes lenguajes artísticos. Esto podría favorecer que el alumnado desarrollara una competencia artística y cultural más en consonancia con lo que es el arte del siglo XXI, un arte caracterizado por la conjunción e integración de diferentes lenguajes expresivos, además de que podría aumentar la calidad de la experiencia estética y a la vez permitir atender diferentes sensibilidades artísticas e individuales.

También se ha constatado un desequilibrio entre las actividades de interpretación, improvisación y composición corporal individual o colectiva. La improvisación estimula la creatividad, la imaginación y la espontaneidad y motiva al alumnado a buscar y expresar un vocabulario corporal propio huyendo de estereotipos y convirtiéndose, por tanto, en una buena herramienta para el desarrollo de la identidad personal. Pero, para que esto sea posible, es clave el grado de musicalidad corporal del maestro o maestra ya que como dice Del Bianco (2009:37): “A través de su propia musicalidad corporal el profesor impulsa la creatividad en el movimiento de los alumnos, aportando su propio vocabulario corporal cada vez que sea necesario". Además, la improvisación es a menudo un camino directo a la composición ya que permite explorar ideas que pueden formar parte de una creación coreográfica posterior. En este sentido, las producciones musicales o artísticas pueden ser una propuesta pedagógica que permita integrar la exploración expresiva, la improvisación y la composición como desarrollo de ideas. Además de posibilitar la integración de diferentes lenguajes y medios artísticos que favorecen el desarrollo de las capacidades creativas y comunicativas del alumnado, son también una herramienta que favorece el trabajo en equipo y la toma de decisiones individuales o colectivas, lo cual puede representar una valiosa contribución al desarrollo de las competencias básicas.

Por último, el estudio pone de manifiesto el valor de consolidar el practicum como el espacio y el proceso en que se activan, desarrollan y evidencian muchas de las competencias profesionales de los futuros y las fu- 
turas maestras, entre las que destacamos el aprendizaje reflexivo sobre la propia práctica y la del resto de profesionales que se pueden observar, la autoevaluación y el compromiso con la propia formación. Además, como dicen Calbó et al. (2009: 41): “... el proceso de las prácticas es un proceso de formación y enriquecimiento mutuo (entre la universidad y la sociedad, la empresa, las instituciones), ya que facilita la conexión entre la teoría explicada en las facultades y la práctica diaria en aspectos de un interés social evidente. Los y las estudiantes aportan las últimas innovaciones que se están explorando en la universidad y los centros de prácticas colaboran en su formación y se tiende a considerarlos agentes de evaluación de esta formación en una medida considerable". Si el valor del trabajo en equipo y de la experiencia compartida impregna la formación inicial de maestros, de alguna manera se puede ver reflejado en su futura actividad docente.

\section{Referencias bibliográficas}

Bachmann, M.L. (1998). La Rítmica Jaques-Dalcroze. Una educación por la música y para la música. Madrid. Piràmide.

Calbó, M. (coord.) et al. (2009). Guia per a l'avaluació de competències en el pràcticum de mestre/a. Agència per a la Qualitat del Sistema Universitari de Catalunya. http:// www.aqu.cat/publicacions/guies_competencies/guia_mestre.html

Coll, C y Onrubia, J. (1999). Discusión entre alumnos e influencia educativa del profesor. Textos didáctica de la lengua y la literatura, n.20, pp.19-37

Del Bianco, S. (2009). El compás, algunos de sus elementos musicales utilizando el movimiento, el espacio y materiales auxiliares. Eufonia, n.47, pp. 33-42.

Garcia, I y cols. (2011) Iniciación a la danza como agente educativo de la expresión corporal en la educación física actual. Aspectos metodológicos. Retos. Nuevas tendencias en Educación Física, Deporte y Recreación, № 20, 33-36pp

Gregorio, V. y Cols. (2010) La danza en el ámbito de educativo. Retos. Nuevas tendencias en Educación Física, Deporte y recreación 2010, № 17, 42-45pp.

Jaques-Dalcroze, E. (1965). Le rythme, la musique et l'éducation. Lausana. Foetisch.

Martin Escobar, M. J. (2005). Del movimiento a la danza en educacion musical. Educatio siglo XXI. Revista de la Facultad de Educación, n 23, 125-140.

Pedrero, C. (2013). Danza en Educacion Primaria. Educatio Siglo XXI, Revista de la Facultad de Educación Vol. 31 n¹ , pp. 129-148

Pérez-Castro,J y Urdampilleta, A . (2012). Expresión Corporal y danza dentro de la Educación Física en las escuelas: propuesta de una unidad didáctica. E.FDeporte.com, Revista Digital. Buenos Aires, Año 17,№ 167.

http://www.efdeportes.com/efd167/expresion-corporal-y-danza-dentro-de-la-educacion-fisica.htm 
Música, movimiento y danza: un enfoque integrador para la formación inicial del profesorado

Silvia Sánchez Ariño, Dolors Cañabate Ortiz, Montserrat Calbó Angrill e Isabel Viscarro Tomás

Pujol, M.A., Serra, J. (1998). La dansa catalana en l'ensenyament primari. Tarragona. El Mèdol i Barcelona. Generalitat de Catalunya. Departament de Cultura.

Rickenmann, R. (2006). Metodologías clínicas de investigación en didácticas y formación del profesorado: un estudio de los dispositivos de formación en alternancia.Actas del Congreso Internacional de Investigación, educación y formación docente, Universidad de Antioquía, Medellín, Colombia, 30 de Agosto a 2 de Septiembre de 2006.

Schubauer-leoni, M.-L. (1998). Les sciences didactiques parmi les sciences de l'éducation: I'étude du projet scientifique de la didactique des mathématiques.- En R. Hofstetter \& B. Schneuwly (Ed.), Le pari des sciences de l'éducation (pp. 329-352). Bruxelles: DeBoeck. 